Vol. 7I, N. ${ }^{\circ}$ II4 (noviembre 20I9), IO7-II7

\title{
TRES PERSPECTIVAS ANALÍTICAS SOBRE LA IDENTIDAD DE GÉNERO Y EL MERCADO DE TRABAJO AGREGADO
}

\author{
THREE ANALYTICAL PERSPECTIVES ON GENDER IDENTITY AND THE ADDED WORK \\ MARKET
}

IRMA GARCÍA-SERRANO

Universidad Central del Ecuador

Recepción manuscrito: 30 de septiembre de 2019

Aceptación versión final: 14 de octubre de 2019

\begin{abstract}
RESUMEN El estudio pretende describir la problemática de la inserción de la variable de género en el mercado laboral desde tres perspectivas analíticas: la ortodoxia neoclásica, la economía feminista y la economía de la identidad, basado en un ejercicio de revisión bibliográfica, para lo cual primero se contextualiza el término género, luego se describe y se reflexiona desde una perspectiva crítica, la evolución de los tres enfoques y en la última sección se concluye la importancia de fomentar y avanzar con la investigación, sobre situaciones relacionadas con la identidad vinculado al género, la etnia, la clase, la edad, a fin de ir sensibilizando a los tomadores de decisiones para la implementación de políticas que disminuyan las brechas en el mercado laboral entre hombres y mujeres.
\end{abstract}

palabras Clave Economía feminista, economía de la identidad, economía neoclásica, mercado de trabajo.

ABSTRACT The study aims to describe the problem of the insertion of the gender variable in the working market from three analytical perspectives: neoclassical orthodoxy, feminist economics and identity economics, based on a bibliographic review exercise, for which the term gender is first contextualized, then it is described and reflected from a critical perspective, the evolution of the three approaches and in the last section it is conclude the importance of promoting and advancing with research on situations related to gender-related identity, the ethnicity, class, age, in order to sensitize decision makers for the implementation of policies that reduce the gaps in the working market between men and women.

KEYWORDS Feminist economics, identity economics, neoclassical economics, labor market.

JEL CODES J16, B13.

\section{INTRODUCCIÓN}

Hoy en día en nuestra sociedad, mujeres y hombres ocupan una posición diferenciada, en la 
mayoría de los casos en condiciones de desigualdad en el acceso a los recursos económicos como el empleo, el reparto de tiempos y trabajos o en el acceso a los espacios de poder, donde se toman las decisiones que afectan la convivencia. Estas condiciones están agudizando los problemas sociales, económicos, ecológicos, éticos y otros aspectos que requieren una reflexión sobre el marco teórico y conceptual que sostiene los comportamientos y valores que acentúan las desigualdades y perjudican el bien común.

Al analizar la convivencia conviene analizar la compleja relación entre los individuos, las colectividades, el Estado y las diversas formas de desigualdad especialmente entre hombres y mujeres dentro del mercado laboral, así como de las relaciones de poder en función del género, la clase, la etnia, la orientación sexual, entre otras.

El estudio pretende describir la variable de género, la cual ha venido a constituirse en un punto de inflexión para el estudio del mercado laboral, pues el trabajo de las mujeres genera impacto en el crecimiento económico y en el desarrollo de la sociedad. En este marco, se intenta responder, en base a la reconstrucción histórico-teórica y en revisión bibliográfica, a la cuestión de ¿por qué los neoclásicos no incluyen en su marco teórico ni distinguen la variable de género en el análisis optimizador de los agentes económicos? y ¿es posible identificar aproximaciones teóricas y prácticas con los estudios de la economía feminista y la economía de la identidad para lograr el reconocimiento del trabajo de las mujeres y además explicar por qué las mujeres resultan discriminadas en categorías laborales?

Desde el pensamiento neoclásico se aprecia que esta escuela no hace distinción del género en el desarrollo de las decisiones de los agentes económicos con carácter general, sin embargo, sí acepta que existe discriminación laboral para determinados grupos sociales entre los que están las mujeres con una mayor dificultad en el proceso de la inserción laboral, principalmente porque dedica más tiempo que el hombre a la familia, lo que ha dado lugar a elaboraciones teóricas de la economía de la familia, donde las diferencias salariales y las condiciones de trabajo por género son analizadas de una manera muy parcial, repercutiendo en las políticas de inversión y en otras áreas como la educación y la formación para el trabajo.

Con respecto al enfoque feminista se observa que esta corriente asume un compromiso político al reconocer el valor de las contribuciones de las mujeres en el ámbito económico. Se analiza el concepto hegemónico de trabajo al criticar la invisibilización del mismo en las esferas de la producción (mercado) y de la reproducción social (ámbito doméstico), y proponer la valoración del trabajo a través de reflejar su aporte en las cuentas nacionales.

Y desde la economía de la identidad, ésta permite ampliar el análisis económico de la variable género como consecuencia de la existencia de normas, costumbres, estereotipos de género y categorías sociales, los cuales constituyen motivos vinculados a la función de utilidad para expresar una amplia gama de gustos y preferencias conforme al contexto social.

\section{MARCO TEÓRICO}

A lo largo de las últimas décadas se ha afianzado el estudio de la desigualdad persistente entre mujeres y hombres, constituyéndose en un tema de análisis entre académicos, políticos y demás investigadores de la ciencia económica, justamente, uno de los temas importantes está 
relacionado con la participación laboral femenina en el mercado de trabajo - trabajo fuera y dentro de los hogares y especialmente el referido a cuidados- y su autonomía económica. Es preciso señalar que el término desigualdad toma vital relevancia en el análisis económico a partir del surgimiento del término género (Bárcena, Prado, Rico y Perez, 2016).

La conceptualización del género ha sido considerada uno de los puntos clave en la teoría feminista desde los años 70, en la medida en que se descubre como una potente herramienta analítica capaz de desvelar las ideologías sexistas ocultas en los textos de las ciencias humanas y sociales (Osborne y Molina, 2008).

El término intenta «reivindicar un territorio definidor específico, de insistir en la insuficiencia de los cuerpos teóricos existentes para explicar la persistente desigualdad entre mujeres y hombres» (Scott, 1996). En palabras de Lagarde (1997), la identidad de género se modifica porque cambia la sociedad y con ello, pueden transformarse los valores, normas y maneras de juzgar los hechos. Gracias al movimiento feminista, se puede diferenciar que el comportamiento económico de hombres y mujeres es diferente como consecuencia de los distintos roles que socialmente se les ha asignado (Carrasco, 2011).

Las diferencias por género en un modelo patriarcal no son neutras, sino que están regidas por la potestad y la subordinación del hombre y la mujer respectivamente, provocan desigualdad y desequilibrio en el acceso a los recursos económicos, incluyendo el empleo, la tierra, los recursos naturales o el crédito (Gálves y Rodríguez, 2012); por lo que para las mujeres la lucha por incorporarse a la vida pública, política y económica y, posteriormente, al mercado de trabajo a través de un empleo remunerado, ha estimulado el interés de los economistas y la reflexión económica acerca de estos aspectos (Castaño, 1999).

Hablar de género ha permitido abordar estudios desde dicha perspectiva clarificando que «perspectiva de género» no significa hacer análisis descriptivos de lo que hacen mujeres y hombres sin investigar en los procesos por qué se producen la desigualdad, la subordinación o la discriminación (Abasolo, Montero, González y Santiago, 2012).

\section{PERSPECTIVA NEOCLÁSICA}

La ortodoxia económica es heredera del pensamiento clásico británico y continuador del modelo capitalista en el desarrollo de la microeconomía del siglo xix. El análisis de los agentes económicos según los criterios de individualidad, racionalidad y maximización es la base del modelo de competencia de un mercado.

El mercado de trabajo es una extensión analítica del modelo clásico que recoge en la oferta de trabajo el comportamiento de los trabajadores y en la demanda de trabajo el comportamiento de las empresas. Para los economistas neoclásicos, el mercado del factor trabajo era considerado de la misma forma que el resto de los mercados de factores de bienes y servicios. Los salarios son el precio que hay que pagar por los servicios prestados por el factor trabajo. Cuanto mayor sean los salarios, menor será la cantidad demandada y mayor la cantidad ofrecida (Perdices de Blas, 2004). Se puede mencionar incluso que esta forma de visualizar guarda concordancia con lo que menciona Gardiner (1999) al argumentar que los marginalistas «sacrificaron el estudio de la actividad económica no monetizada en aras de hacer de la economía una ciencia “exacta” (Pérez Orozco, 2006). 
Posteriormente, la denominada nueva economía de la familia, expresión de la teoría neoclásica para el estudio del comportamiento de la familia, fundamentado en la obra Tratado sobre la familia, de Gary Becker (1981), aplica la teoría de las ventajas comparativas. Según estas ventajas, un hogar eficiente es aquel donde cada miembro se va a especializar en el hogar o en el mercado conforme tenga mayores ventajas comparativas, y una vez especializado, cada miembro invertirá únicamente capital humano para el mercado o capital humano para lo doméstico, según se haya especializado en uno o en otro. Es decir, que al interior de la familia se produce un equilibrio automático en la distribución del tiempo y los beneficios (Anzorena, 2009).

En relación con el factor trabajo, el estudio se realiza desde dos ámbitos bien diferenciados: el trabajo productivo y el trabajo reproductivo; el primero como destinado a lo público, a la representación social a la participación política, por lo general con roles asignados para los hombres; y el segundo, a lo doméstico y al mantenimiento y preservación de la vida, con roles asignados solo a las mujeres. Pero resulta que estos dos ámbitos no tienen la misma valoración social, dando lugar a la exclusión y desigualdad histórica entre mujeres y hombres (Brunet y Santamaría Velasco, 2015).

\section{PERSPECTIVA FEMINISTA}

El término de economía feminista se ha extendido para referirse a un conjunto, difusamente delimitado, de análisis relativos al género y la economía y a pesar de la marginación académica y política que sufre todo análisis relativo al género, está logrando posicionarse dentro del pensamiento económico (Perez Orozco, 2005a).

La economía feminista devela análisis económicos críticos sobre las diferencias entre mujeres y hombres (Pérez, 2006). Para continuar, conviene realizar una breve diferenciación entre economía de género caracterizada por considerar la apertura del proceso científico a un nuevo sujeto (femenino) eliminando los sesgos androcéntricos; y, por otro lado, la economía feminista caracterizada por sus análisis de la «exclusión de las mujeres» criticando que no ha sido una mera consecuencia de la mala aplicación del método científico, sino que el método mismo y los criterios que lo han guiado encierran sesgos (Pérez, 2006).

Esta visión crítica reformula el concepto de trabajo hegemónico que, en su generalidad, invisibiliza una parte fundamental del trabajo necesario para cubrir las necesidades humanas: el trabajo de cuidados. En palabras de la misma autora (Pérez, 2006), el trabajo de cuidados está en crisis, su propia definición sigue en continuos debates, solo puede percibirse en toda su magnitud si dejamos de centrar la visión en los mercados y lo monetizado y en cambio lo situamos como categoría analítica básica «la sostenibilidad de la vida» (Pérez, 2006).

La economía feminista, por un lado, trata de romper con las limitaciones de la noción de trabajo doméstico e incorpora una visión multilateral que entrelaza lo material-corporal y lo afectivo-relacional y, por otro lado, plantea la creación de una cuenta satélite para estudiar el trabajo no remunerado, es decir, visualiza y cuantifica el trabajo doméstico y es lo que se conoce como la economía feminista de la conciliación; sin embargo, merece en este marco mencionarse a la economía feminista de la ruptura, la cual cuestiona en cierta medida algunas concepciones de conceptos y metodologías anteriores y coloca en el centro del análisis - la 
sostenibilidad de la vida- y además intenta diferenciar las relaciones de poder entre las propias mujeres (Pérez Orozco, 2005).

Con lo anotado, se puede mencionar que los elementos definitorios de esta corriente aún están indefinidos, siguen en un continuo debate y bajo este concepto se agrupan propuestas de muy diversa índole que valen la pena continuar investigando.

\section{ECONOMÍA DE LA IDENTIDAD}

El pensamiento económico dominante ha sido desafiado por nuevos desarrollos teóricos, traspasando aquellos fines solo relacionados a preguntas como el ingreso y el consumo, uno de esos desarrollos es la economía de la identidad analizado por George Akerlof — premio Nobel de economía- y Rachel Kranton con su Economía de la identidad (Akerlof, George y Krantom, 2000).

Los autores explican cómo la identidad, el sentido de sí mismo de una persona, afecta los resultados económicos, pues la «identidad o las identidades y las normas se derivan del entorno social» (Akerlof, 2010), se asocian con diferentes categorías sociales y definen de alguna manera como deben comportarse las personas en estas categorías, también indican que la identidad puede explicar muchos fenómenos como el conflicto étnico y racial, la discriminación, las disputas laborales intratables entre otros, coincidiendo con lo que mencionan en su frase «nuestras identidades dan forma a nuestro trabajo, los salarios y el bienestar» (Akerlof, 2010).

En este mismo sentido, se menciona que las mujeres obtienen un menor salario promedio que los hombres y no es solo por la discriminación de género, sino que obedece a estereotipos de cómo deben comportarse cada género ante situaciones sociales, igual situación sucede a la hora de la negociación salarial, las mujeres contraofertan menos que los hombres, ya que los hombres negocian de manera más agresiva en empresas con presencia de mujeres (Gómez, Vázquez, Cortina y Grajales, 2018), o la feminización del profesorado de educación primaria ha coincidido con una baja en el estatus social de esa ocupación, así como en su retribución relativa (Fernández, 2013), asimismo, la ocupación de cuidados es vista como un status inferior.

Siguiendo la misma línea Claudia Goldin, con su pollution theory of discrimination, muestra que, a veces, en el caso de una ocupación muy masculinizada, la entrada de las primeras mujeres en la misma se percibe como una amenaza - a la identidad, al estatus- de los hombres, igual que la ayuda de políticas de afirmación positiva (cuotas) que no les gustan a algunas personas porque habrá la sospecha que ha llegado a directivas como consecuencia de la cuota y no por sus propios méritos, lo que puede acarrear conductas hostiles de algunos de ellos hacia sus nuevas compañeras o mujeres que serán juzgadas negativamente (Escot, Fernández y Arellano, 2013).

\section{DISCUSIÓN}

El enfoque neoclásico no ayuda a reformar el análisis económico, así como el estudio del mercado laboral con categorías económicas generales no podría introducir el debate sobre las relaciones de género que están involucradas en la producción. Su limitación teórica genera estudios con menos riqueza analítica, por ejemplo, en Nicaragua (1991-1993), cuando el Estado 
retiró apoyo a las cooperativas de producción (Utting, Chamorro y Bacon, 2017) se intensificaron las discusiones en torno a los derechos de sus miembros. Los varones argumentaron que las mujeres no eran tan productivas como los hombres y que perdían demasiados días de trabajo debido a enfermedades de sus hijos. La licencia de maternidad también se convirtió en fuente de disputas, debido a que muchas veces los contratantes suponen que el costo del pago a una mujer resulta mayor que si pagaras a un hombre y por el tiempo de permiso que varía entre países. Dorien Brunt (1995) comenta que en la región de Jalapa, donde ella investigó, de muchas maneras se les hizo saber a las mujeres que ya no eran útiles para la cooperativa (Deere, 2004). Los estudios evidencian un hecho económico formal, sin embargo, el imaginario y las políticas siguen acentuando diferencias entre mujeres y hombres con un evidente sesgo de género que deja oculto o invisibiliza todo lo que no entre en su marco teórico. Es decir, en esta perspectiva de análisis, difícilmente podríamos encontrar alguna diferencia entre el trabajo que realizan las mujeres y el trabajo que realizan los hombres (Pérez Orozco, 2005).

Igualmente, desde la economía de la familia, Becker sistematiza aquello que en las sociedades se asume como dado: que los hogares compuestos por varones (padres) proveedores y mujeres (madres) cuidadoras son más eficientes que los hogares donde no están ambos miembros (Anzorena, 2009). Con lo que se puede concluir que todo hogar diferente a la familia heterosexual patriarcal sería ineficiente. Además, la teoría del capital humano, como ampliación del modelo neoclásico es completamente deductiva e insuficiente para el estudio de la participación laboral femenina. La familia es visualizada como una unidad económica que es parte del mercado capitalista, donde, las mujeres trabajan sin remuneración (Mangas, Andrés y Cornejo, 2005).

Desde el punto de vista de la microeconomía, uno los principales postulados neoclásicos, es el estudio de la productividad marginal el mismo que proporciona cierta justificación de la distribución del ingreso existente, ya que, al remunerar a cada factor respecto a su productividad marginal, se obviaba el concepto de plusvalía y se eliminaba todo signo de explotación del trabajo y, por tanto, creían en la eficiencia de los mercados (Perdices de Blas, 2004). Entendiéndose que para esta perspectiva la desigualdad en los ingresos de las y los asalariados, es explicada por las diferencias en sus niveles de productividad y ésta, a su vez, se presenta por la diferencia de la participación de las mujeres solamente en ciertas actividades económicas (Mangas et al., 2005).

La economía feminista fue muy crítica tanto con el enfoque neoclásico como con las nociones de la nueva economía de la familia, por mencionar a alguna autora, encontramos a Antonella Picchio, quien argumenta que «la idea de la sustitución sistemática entre trabajo doméstico y trabajo asalariado es insostenible, cuando en los hechos se comprueba que las mujeres acumulan y soportan una carga global de trabajo más alta que los varones porque los procesos de producción y reproducción, implican tiempos, controles y relaciones de fuerzas no homogéneos, aspectos que no puede recogerse en un análisis metodológico atemporal y ahistórico (Anzorena, 2009).

Pérez Orozco, resalta la participación de la mujer en la economía, reconoce a las mujeres la condición de sujeto epistemológico capaz de crear conocimiento (2006). También otros autores (Mangas et al., 2005; Anzorena, 2009) critican a la economía familiar por sus clásicos 

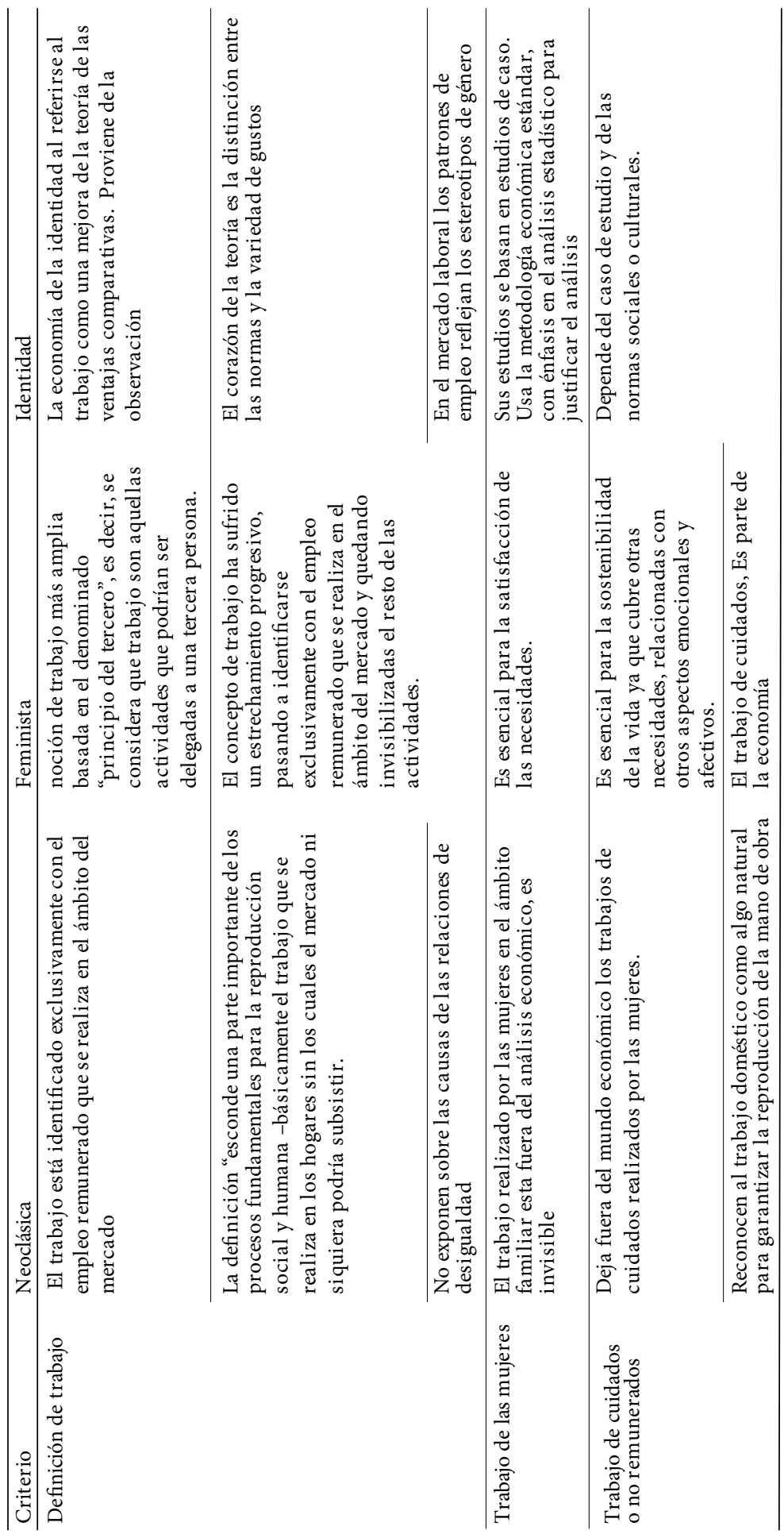

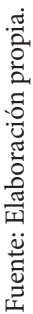


planteamientos de supuesta neutralidad del sistema económico y por su justificación en la creación y mantenimiento de trabajos mal remunerados.

Por tanto, la segregación ocupacional genera ventajas para los hombres y desventajas para las mujeres, como resultado de prejuicios y estereotipos de género, en el tema salarial existen brechas donde a igual trabajo las mujeres reciben menos paga, los empleadores tienen preferencias para ocupar en algunas posiciones a los hombres y en otras a las mujeres; según el autor Saraví (1997), la concentración del trabajo femenino en un conjunto restringido de actividades afecta sus niveles de productividad convirtiéndose éste en un factor que explica las diferencias salariales.

Por otro lado, la economía feminista pretende demostrar la desigualdad, ha insertado al debate que el trabajo de los cuidados es un elemento clave para la sostenibilidad de la vida en su extensión humana, social y económica. Además, su defensa ha incidido en los gobiernos para poner asunto en la necesidad de promover el rol productivo de las mujeres como forma de impulsar el desarrollo a través de la investigación, creación de metodologías, la aplicación de herramientas como la encuesta del uso del tiempo, la introducción de las cuentas satélites y, sobre todo, por incluir en los estudios la variable de género (Carrasco, 2015).

Son las propias mujeres las que han contribuido a develar que las estructuras económicas y sociales existentes, son las que subordinan a las mujeres por razón de sexo en los mercados laborales, permitiendo que persistan mujeres que reciben un menor salario por el mismo trabajo, menos cantidad mujeres en cargos gerenciales, más mujeres profesionales con mejores calificaciones y sin acceso al trabajo.

Finalmente, sobre la economía de la identidad, los científicos sociales en otras disciplinas la encuentran útil, ya que conecta los modelos económicos con su propio trabajo, lo que permite un desarrollo más rico de los procesos sociales. Esta perspectiva intermedia complementaria con la ortodoxia devela que estrategas de negocios, analistas, políticos pueden beneficiarse de la economía de identidad, ya que ofrece formas de predecir con mayor precisión las consecuencias de las políticas públicas y las prácticas comerciales.

Es decir, en este caso la perspectiva de la economía de la identidad pretende desmenuzar y realizar un análisis a grupos más específicos para contribuir a identificar particularidades de acuerdo con su contexto social, cultural, económico. Por ejemplo, cuando se analiza la problemática especialmente de las mujeres indígenas y afro aparecen dos dimensiones claramente: etnicidad y género.

Sin embargo, a la fecha existen pocos trabajos que logren explicar cómo se construyen las relaciones de género y como se conceptualiza con los sistemas de creencias y valores de acuerdo con cada contexto. Es decir, ha resultado más difícil vincular la identidad genérica y étnica, por lo que no ha habido un uso consistente de la variable identidad, no se ha aclarado la relación entre identidad, cultura, cosmovisión, ni se define si se trata de un proceso individual o pasa a lo colectivo y faltan elementos metodológicos que ayuden a investigar esta variable, por lo que ésta es una línea que demanda mayores investigaciones futuras (Judith et al., 2000).

Por otra parte, debido a su poder explicativo, numerosos académicos en psicología, sociología, ciencias políticas, antropología e historia han adoptado la identidad como concepto 
central que puede ser llevada al análisis económico, permitiendo una nueva visión de muchos problemas socioeconómicos (Akerlof, 2000).

Las funciones de utilidad se han desarrollado para expresar una amplia gama de gustos y preferencias no pecuniarias, como el deseo de tener hijos, la preocupación por el Estado y el deseo de justicia y retribución en la que la economía de la identidad permite mejorar la explicación. La identidad en sí misma puede ser una opción. Hasta cierto punto, la gente puede elegir a quién o qué quieren ser. Un ejemplo es la elección que se enfrenta una mujer de clase media entre una carrera y convertirse en un ama de casa.

Al final se incluye una tabla (ver Tabla 1) que identifica los desencuentros relevantes, de algunos términos relacionados con la variable género y el mercado de trabajo desde las perspectivas estudiadas.

\section{CONCLUSIONES}

La perspectiva de la ortodoxia no incluye la variable de género en su análisis, por lo que no aporta en el análisis teórico para explicar las desigualdades que se presentan en el mercado de trabajo; sus resultados se basan en estudios de tipo cuantitativo, pocos de tipo cualitativo, que no contribuyen a un avance en la discusión para reducir las desigualdades.

El enfoque neoclásico no tiene interés en el análisis de la oferta y la demanda de trabajo femenino, no reconoce el trabajo doméstico y afecta directamente la valoración del trabajo de la mujer.

La economía feminista es una propuesta que ha logrado visualizar la variable de género en la academia, recuperar su condición de sujeto que puede crear conocimiento económico, ha permitido reconocerla como un actor protagonista de la vida social y económica de los países. Ha desarrollado opciones críticas de oposición al patriarcado, ha contribuido a crear metodologías y la valorización del trabajo de cuidados para intentar trasparentar las cuentas nacionales en varios países. El tema está en los discursos tanto políticos como económicos y abordado por varias disciplinas de las ciencias.

La economía de la identidad amplía el foco del análisis de género dentro de un marco intermedio y más complementario con la economía neoclásica y está tomando muchas direcciones; su análisis sobre la variable de género da cuenta de que las normas y las instituciones interfieren para que las mujeres puedan cambiar la situación de desigualdad que persiste. Igualmente, brinda la oportunidad para abrir mayores líneas de investigación para estudiar la relación entre identidad étnica, cultura y cosmovisión o cómo se construyen las relaciones entre los géneros y cómo se contextualizan con los sistemas de valores y creencias de los propios grupos - sean por etnia, por edad- y traspasa a estudios sobre migración, educación entre otros, al decir que el género y la raza son solo las manifestaciones más claras de la identidad y las normas.

Visto desde cualquier enfoque de análisis la variable identidad de género, concretamente la oferta del trabajo femenino en el mercado laboral, continúa siendo invisibilizada cuando no se reconoce el aporte social y económico del trabajo doméstico a las economías de los países. Esta situación, además, genera brechas injustas en la distribución de las actividades, especialmente las de cuidado, limita la mejora en la participación de mujeres en el mercado laboral y cuando lo ha logrado debe invertir mayores esfuerzos para mantenerse. 
Por lo antes referido, es indispensable ampliar el debate, cambiar los paradigmas androcéntricos de la economía y el trabajo, superar criterios económicos y de optimización en los estudios y considerar otros análisis como feminismo descolonial en América Latina y categorías como la interseccionalidad de género en futuras investigaciones.

\section{REFERENCIAS}

Abasolo, O., Montero, J., González, H. y Santiago, B. (2012). Trabajos: empleo, cuidados y división sexual del trabajo. Guía Didáctica de Ciudadanía con perspectiva de género. Igualdad en la diversidad para profesorado de Segunda Etapa Eso y Bachillerato. Recuperado de http://www.fuhem.es/ proyecto_igualdad/pdf/cap_3.pdf

Akerlof, G. and Krantom, R. (200o). Economics and Identity. Oxford Journals, 115(3), 715-753.

Akerlof, G. and Krantom, R. (2010). Identity Economics. Princeton, USA: Princeton Editorial Associates, Inc. Anzorena, C. (2009). El ¿retorno? del Tratado sobre la Familia de Gary Becker. Algunas reflexiones en torno a los criterios de eficiencia que legitiman los planes compensatorios. KAIros Revista de Temas Sociales, 13(24), 1-15. Recuperado de http://philpapers.org/rec/ANZERD

Arceo Gómez, E., Campos Vázquez, R., Medina Cortina, E. y Vélez Grajales, R. (2018). Negociación y preferencias económicas por género : evidencia experimental en México. El Trimestre Económico, $\operatorname{LXXXV}(3), 645-678$.

Bárcena, A., Prado, A., Rico, M. y Pérez, R. (2016). Autonomía de las mujeres e igualdad en la agenda de desarrollo sostenible. IEEE Transactions on Automatic Control. https://doi.org/10.1109/ TAC.1975.1101110

Brunet, I. y Santamaría Velasco, C. (2015). La economía feminista y la división sexual del trabajo. Culturales, Iv(1), 61-86. Recuperado de http://www.scielo.org.mx/pdf/cultural/v4n1/2448-539Xcultural-4-01-0oo61.pdf

Carrasco, C. (2011). La economía del cuidado: planteamiento actual y desafíos pendientes. Revista de Economía Crítica, (11), 205-225.

Carrasco, C. (2015). El tiempo más allá del reloj: las encuestas de uso del tiempo revisitadas. Cuadernos de Relaciones Laborales, 34(2), 357-383. Recuperado de https://doi.org/10.1038/nbt.218o

Castaño, C. (1999). Economía y género. Política y Sociedad, 32(23). Recuperado de https://doi.org/10.5209/ POSO.25802

Deere, C. y León M. (2004, agosto). Revertir la exclusión de género: Lecciones a partir de América Latina. El otro Derecho, (31-32), 2-40.

Escot Mangas, L. y Fernández Cornejo, J. A. (coords.). (2013). Análisis de algunas de las causas últimas de la desigualdad de género en el mercado laboral. Implicaciones para las políticas de empleo. Recuperado de https://www.ucm.es/data/cont/docs/85-2013-12-17-Causas\%20\%C3\%BAltimas\%20 desigualdad\%20g\%C3\%A9nero\%2027_septiembre_2013(corregido).pdf

Escot Mangas, L., Fernández Cornejo, J. A. y Poza Lara, C. (2005). La división del trabajo entre los miembros de la pareja: evolución, ideas y fundamentos teóricos, 741-754. Recuperado de https://www. ucm.es/data/cont/docs/85-2013-11-29-divisiontrabpareja.pdf

Gálves, L. y Rodríguez, P. (2012). La desigualdad de género en las crisis económicas. Investigaciones Feministas, (2), 113-132. Recuperado de https://doi.org/10.5209/rev_INFE.2011.v2.38607 
Osborne, R. y Molina, C. (2008). Evolución del concepto de género. Revista de Metodología de Ciencias Sociales, 15(15), 147-182.

Perdices de Blas, L. (ed.). (2004). Historia del pensamiento económico. Madrid, España: Síntesis Ed.

Pérez Orozco, A. (2005a). Economía del género y economía feminista ¿conciliación o ruptura? Revista Venezolana de Estudios de La Mujer, 10(24), 43-64. Recuperado de http://187.216.193.232/biblosimdf/sites/default/files/archivos/o0353Econgenyfem.pdf

Pérez Orozco, A. (2005b). Economía del género y economía feminista ¿Conciliación o ruptura? Revista Venezolana de Estudios de la Mujer, $10(22)$.

Pérez Orozco, A. (2006a). Amenaza tormenta. La crisis de los cuidados y la reorganización del sistema. Recuperado de http://revistaeconomiacritica.org/sites/default/files/revistas/n5/1_amenaza_tormenta.pdf.

Pérez Orozco, A. (2006b). Perspectivas feministas en torno a la economía: el caso de los cuidados. Recuperado de https://doi.org/10.1017/СвO9781107415324.004

Sánchez Gómez M. J. y Goldsmith, M. (200o). Reflexiones en torno a la identidad étnica y genérica. Estudios sobre las mujeres indígenas en México. Política y Cultura, (14), 61-88. Recuperado de https://www.redalyc.org/articulo.oa?id=26701404

Scott, J. W. (1996). El género : una categoría útil para el análisis histórico. https://doi.org/10.2307/1864376 Utting, P., Chamorro, A. y Bacon, C. (2017). El rol de las cooperativas y la acción colectiva en el desarrollo y la reconciliación en Nicaragua, (30), (s. d.). 